\title{
Quelques méthodes probabilistes pour les équations aux dérivées partielles
}

\author{
Étienne Pardoux
}

Résumé. Après une introduction qui indique le lien entre opérateurs aux dérivées partielles du second ordre et processus de diffusion, et présente plusieurs approches probabilistes récentes des EDP non linéaires, nous introduisons les "équations différentielles stochastiques rétrogrades"-EDSR, et leurs liens avec les systèmes d'EDP semilinéaires du second ordre et leur solution de viscosité. Nous donnons l'essentiel des démonstrations des principaux résultats. Enfin nous énonçons trois résultats qui ont été établis récemment à l'aide des EDSR, l'un dû à Pradeilles traite de la propagation des fronts dans les équations de KPP généralisées, les deux autres dûs respectivement à Buckdahn, Hu, Peng et à l'auteur traitent de l'homogénéisation d'EDP semilinéaires à coefficients périodiques.

Mots clés. Mouvement brownien, Equation différentielles stochastiques, Equations différentielles stochastiques rétrogrades, Solutions de viscosité de systèmes d'EDP, Homogénéisation d'EDP semi-linéaires.

\begin{abstract}
After an introduction which presents the relation between second order partial differential operators and diffusion processes, and several recent probabilistic approaches to nonlinear PDEs, we introduce the notion of "backward stochastic differential equations" -BSDEs, and their connection with viscosity solutions of systems of semilinear second order PDEs. We give most of the essential proofs. Finally, we describe three results on nonlinear PDEs which have been recently established with the help of BSDEs. One, due to Pradeilles, describes wave front propagation for generalized KPP equations. The two others, due respectiveley to Buckdahn, Hu, Peng and to the author, study the homogenization of semilinear PDEs with periodic coefficients.
\end{abstract}

Keywords. Brownian motion, Stochastic differential equations, Backward stochastic differential equations, Viscosity solutions of systems of PDEs, Homogenization of semilinear PDEs.

AMS Subject Classification. 60H30,60J60,35B27,35C99,35D05,35K57.

\section{Introduction}

Le but de cet article est de montrer le lien entre les équations aux dérivées partielles du second ordre, et les processus stochastiques de diffusion, ainsi que de présenter quelques Article published by EDP Sciences and available at http://www.edpsciences.org/proc or http://dx.doi.org/10.1051/proc:1999048 
résultats obtenus récemment sur des équations aux dérivées partielles linéaires et non linéaires, par des méthodes probabilistes.

Les liens entre processus de diffusion et équations qux dérivées partielles du second ordre sont au centre de travaux de Kolmogorov des années 1930. En fait, beaucoup de solutions d'EDP linéaires du second ordre peuvent s'écrire comme l'espérance d'une fonctionnelle d'un processus de diffusion. Donnons une telle formule dans un cas très simple. Soit $D$ un domaine borné de $\mathbb{R}^{d}$ de frontière régulière, $f \in C(\partial D)$. Alors la solution $u$ du problème de Dirichlet

$$
\begin{aligned}
\frac{1}{2} \Delta u(x) & =0, \quad x \in D \\
u(x) & =f(x), \quad x \in \partial D
\end{aligned}
$$

est donnée par la formule

$$
u(x)=\mathbb{E}\left[f\left(B_{\tau_{x}}^{x}\right)\right],
$$

où $\left\{B_{t}^{x} ; t \geq 0\right\}$ désigne le mouvement brownien "standard" à valeurs dans $\mathbb{R}^{d}$ partant de $x$ à l'instant 0 , et $\tau_{x}$ est l'instant (aléatoire) où ce processus sort du domaine $D$.

Si l'on complique un peu l'équation, sous la forme

$$
\begin{aligned}
\frac{1}{2} \Delta u(x)+c(x) u(x)+g(x) & =0, \quad x \in D, \\
u(x) & =f(x), \quad x \in \partial D,
\end{aligned}
$$

la formule devient

$$
u(x)=\mathbb{E}\left[f\left(B_{\tau_{x}}^{x}\right) \exp \left[\int_{0}^{\tau_{x}} c\left(B_{t}^{x}\right) d t\right]+\int_{0}^{\tau_{x}} g\left(B_{t}^{x}\right) \exp \left[\int_{0}^{t} c\left(B_{s}^{x}\right) d s\right] d t\right] .
$$

On a des formules analogues pour d'autres types de condition au bord, par exemple pour des conditions de type Neuman, et pour des équations paraboliques.

Si l'on remplace l'opérateur $\frac{1}{2} \Delta$ par un opérateur plus général du type

$$
L=\frac{1}{2} \sum_{i, j=1}^{d} a_{i, j}(x) \frac{\partial}{\partial x_{i}} \frac{\partial}{\partial x_{j}}+\sum_{i=1}^{d} b_{i}(x) \frac{\partial}{\partial x_{i}},
$$

le mouvement brownien est remplacé par un processus de diffusion de générateur infinitésimal $L$, i.e. par la solution de l'équation différentielle stochastique ("EDS")

$$
\begin{aligned}
d X_{t} & =b\left(X_{t}\right) d t+\sigma\left(X_{t}\right) d B_{t} \\
X_{0} & =x,
\end{aligned}
$$

où $\sigma(x)$ est une racine carrée de la matrice $a(x)$. Dans le cas où le processus sous-jacent est récurrent positif et ergodique, avec $\mu$ comme unique probabilité invariante, on peut étudier l'EDP

$$
L u(x)+g(x)=0, \quad x \in \mathbb{R}^{d},
$$


pourvu que $\int g(x) \mu(d x)=0$. On peut alors étudier le comportement à l'infini de $u$, étant donné celui de $g$, cf. la section 2 ci-dessous.

Dans le cas d'un opérateur du second ordre sous forme divergence, qui par faute de régularité des coefficients $a_{i, j}$ ne peut pas se réécrire sous la forme ci-dessus, on peut encore définir un processus de Markov associé $\left\{X_{t} ; t \geq 0\right\}$, mais on ne peut pas toujours écrire l'EDS dont il serait la solution, et on ne dispose pas des mêmes outils probabilistes pour le manipuler.

Les formules probabilistes du type ci-dessus, souvent appelées formules de FeynmanKac, constituent un outil qui permet de démontrer de nombreux résultats sur l'EDP correspondante par des méthodes probabilistes. Elles sont l'objet de très nombreux travaux, et nous renvoyons le lecteur entre autres aux livres [2], [3], [5], [9], [16], [17], [20], [34].

Signalons enfin que l'on a une interprétation probabiliste pour d'autres types d'équations aux dérivées partielles, appelées équations de Fokker-Planck. Si $\left\{X_{t} ; t \geq 0\right\}$ est la solution de l'EDS ci-dessus, $L$ est son générateur infinitésimal, ce qui signifie en particulier que pour tout $f \in C_{c}^{\infty}\left(\mathbb{R}^{d}\right)$,

$$
\frac{d}{d t} \mathbb{E}\left[f\left(X_{t}\right)\right]=\mathbb{E}\left[L f\left(X_{t}\right)\right]
$$

Désignons par $\mu_{t}$ la loi de probabilité de $X_{t}$, pour tout $t \geq 0$. Alors la dernière égalité se réécrit :

$$
\frac{d}{d t}<\mu_{t}, f>=<\mu_{t}, L f>
$$

soit, en tout cas au sens des distributions,

$$
\frac{d \mu_{t}}{d t}=L^{*} \mu_{t}
$$

Si pour tout $t>0, \mu_{t}(d x)$ possède la densité $p(t, x)$ par rapport à la mesure de Lebesgue, et si par exemple pour tout $1 \leq i \leq d$,

$$
\sum_{j=1}^{d} \frac{\partial a_{i j}}{\partial x_{j}} \in L^{\infty}\left(\mathbb{R}^{d}\right)
$$

alors l'équation pour l'évolution de la densité de $X_{t} s$ 'écrit :

$$
\frac{\partial p}{\partial t}(t, x)=\frac{1}{2} \sum_{i, j=1}^{d} \frac{\partial}{\partial x_{j}}\left(a_{i j} \frac{\partial p}{\partial x_{i}}(t, \cdot)\right)(x)+\sum_{i=1}^{d} \frac{\partial}{\partial x_{i}}\left(\bar{b}_{i} p(t, \cdot)\right)(x),
$$

avec $\bar{b}_{i}=\frac{1}{2} \sum_{j=1}^{d} \frac{\partial a_{i j}}{\partial x_{j}}-b_{i}$.

On trouvera dans [20] des formules probabilistes pour des solutions d'EDP "de type Fokker-Planck". 
En outre, on dispose maintenant de plusieurs généralisations de la formule de Feynman-Kac à des EDP nonlinéaires.

La première, et probablement la plus ancienne, consiste à remplacer l'EDS ci-dessus par une EDS contrôlée, i.e. où les coefficients $b$ et $\sigma$ dépendent d'une fonction de $t$ et de $x$ à choisir sous certaines contraintes, de façon à minimiser un certain coût. La fonction de valeur $u(t, x)$ du problème de contrôle, où $u(t, x)$ représente le cô̂t minimal à partir de l'instant $t$, quand le processus contrôlé part à cet instant du point $x$, est la solution d'une EDP non linéaire. Nous renvoyons le lecteur intéressé aux ouvrages [5], [6] et [15].

La seconde généralisation consiste à faire dépendre les coefficients de l'EDS non seulement de la position $X_{t}$ du processus à l'instant $t$, mais aussi de sa loi de probabilité $\mu_{t}$. On construit alors ce que l'on appelle un "processus de diffusion non linéaire", auquel on peut associer 1'EDP quasi-linéaire de Fokker-Planck qui gouverne l'évolution de la loi de probabilité de $X_{t}$. Un tel type de processus a été semble-t-il pour la première fois introduit par MacKean [24].

Par exemple, l'équation de Boltzmann est une équation de Fokker-Planck pour un processus de Markov non linéaire, cf. Méléard [25], Sznitman [37]. Cette interprétation probabiliste donne une intuition sur cette équation différente des méthodes analytiques. Elle permet notamment de considérer des conditions initiales de type mesure, et d'établir des résultats de régularité en s'appuyant sur la calcul de Malliavin pour les processus de saut. Enfin, elle est à la base des méthodes numériques de type "méthodes particulaires stochastiques". Par exemple, en discrétisant le temps non pas à des instants fixes, mais aux instants de saut d'un processus de Poisson sous-jacent, on contrôle mieux la vitesse de convergence pour certains types d'équations de Boltzmann, cf. Desvillettes, Graham, Méléard [10].

Beaucoup d'équations aux dérivées partielles d'évolution non linéaires s'écrivent comme des équations de Fokker-Planck, comme par exemple l'équation des milieux poreux, cf. en particulier Benachour, Chassaing, Roynette, Vallois [4]. Une autre équation qui šinterprète de la même façon est celle satisfaite par le le tourbillon, i.e. le rotationnel de la solution de l'équation de Navier-Stokes bidimensionnelle. Cette remarque a permis à Méléard [26] de démontrer la convergence de la méthode numérique des vortex de Chorin. Pour une interprétation probabiliste de l'équation de Navier-Stokes tridimensionnelle, on pourra consulter Le Jan, Sznitman [23].

La troisième généralisation consiste à remplacer le processus de diffusion par un processus de diffusion-branchement, ou un "super-processus", qui est un processus à valeurs mesure, auquel on peut associer certaines EDP semi-linéaires. Ces processus et leurs liens avec les EDP ont fait l'objet de nombreux travaux ces dernières années, cf. en particulier [11], [12], [21], [22].

Citons deux résultats sur l'EDP elliptique non linéaire

$$
\Delta u=u^{2} .
$$

démontrés récemment par des méthodes probabilistes. D'une part, Le Gall [21] et Dynkin, Kuznetsov [13] ont montré que l'ensemble des solutions positives $u$ de cette équation dans 
un ouvert régulier $D$ est en bijection avec les conditions aux bords, lequelles peuvent être essentiellement spécifiées par la partie du bord où $u$ vaut $+\infty$, et une mesure positive sur l'autre partie du bord. Enfin Dhersin, Le Gall [11] ont établi un test pour qu'un point du bord soit "régulier" pour cette équation.

Enfin, la quatrième consiste à associer à la diffusion $X_{t}$ un couple de processus $(Y, Z)$, solution de ce que l'on appelle improprement une "équation différentielle stochastique rétrograde", et que l'on devrait plutôt appeler un problème inverse pour une EDS.

La théorie des EDSR, et leur lien avec les systèmes d'EDP semi-linéaires sera présentée à la section 3 . Les sections 4 et 5 présentent des résultats asymptotiques sur les EDP, qui ont été démontrés récemment à l'aide des EDSR.

\section{2 Équations de Poisson}

Le but de cette section est de présenter des résultats de Pardoux, Veretennikov [32] concernant l'équation de Poisson dans $\mathbb{R}^{d}$ :

$$
L u(x)+f(x)=0, x \in \mathbb{R}^{d} ;
$$

où $L$ est un opérateur elliptique du second ordre :

$$
L=\frac{1}{2} \sum_{i, j=1}^{d}\left(\sigma \sigma^{*}\right)_{i j}(x) \frac{\partial^{2}}{\partial x_{i} \partial x_{j}}+\sum_{i=1}^{d} b_{i}(x) \frac{\partial}{\partial x_{i}}
$$

dont les coefficients satisfont des hypothèses qui seront précisées ci-dessous, et $f$ satisfait une condition de "centrage". L'interprétation probabiliste est la suivante. Soit $\left\{X_{t} ; t \geq 0\right\}$ la solution de l'équation différentielle stochastique - EDS :

$$
X_{t}=x+\int_{0}^{t} b\left(X_{s}\right) d s+\int_{0}^{t} \sigma\left(X_{s}\right) d B_{s}, t \geq 0 .
$$

Sous les conditions ci-dessous, $\left\{X_{t}\right\}$ possède une unique mesure de probabilité invariante

$\mu$ (et on suppose que $\int f(x) \mu(d x)=0$ ). La solution de l'équation de Poisson (1) est donnée par la formule

$$
u(x)=\int_{0}^{\infty} \mathbb{E}_{x} f\left(X_{t}\right) d t
$$

où $\mathbb{E}_{x}$ désigne l'espérance partant de $x$, i.e. avec la condition initiale $X_{0}=x$. L'objet de [32] est de montrer que la formule (2) fournit une solution à l'équation (1), qui est unique dans une certaine classe, et en outre que si $f$ croît (ou décroît) au plus comme un certain polynôme à l'infini, $u$ est borné ou bien croît au plus polynômialement à l'infini.

On suppose qu'il existe $\lambda_{-}, \lambda_{+}$et $\Lambda$ tels que pour tout $x \in \mathbb{R}^{d} \backslash\{0\}$,

$$
0<\lambda_{-} \leq<\sigma \sigma^{*}(x) \frac{x}{|x|}, \frac{x}{|x|}>\leq \lambda_{+}, \operatorname{Tr} \sigma \sigma^{*}(x) \leq \Lambda d .
$$


On suppose en outre qu'il existe $\alpha \geq-1, r>0$, et $M>0$ (dans le cas $\alpha=-1$, on impose que $\left.2 r>3 \lambda_{+}-\lambda_{-}+\Lambda d\right)$ tels que pour tout $|x|>M$,

$$
<b(x), \frac{x}{|x|}>\leq-r|x|^{\alpha} .
$$

On montre (cf. Veretennikov [38]) que sous les hypothèses ci-dessus, le processus $\left\{X_{t} ; t \geq 0\right\}$ possède une unique mesure de probabilité invariante $\mu$, telle que si $\mu^{x}(t)$ désigne la loi de $X_{t}$ lorsque $X_{0}=x, \operatorname{var}(\cdot)$ la norme de la variation totale,

Lemme 2.1 Pour $2 k+2<m, m$ assez petit dans le cas $\alpha=-1$, il existe $C$ tel que

$$
\operatorname{var}\left(\mu^{x}(t)-\mu\right) \leq C\left(1+|x|^{m}\right)(1+t)^{-(k+1)}, x \in \mathbb{R}^{d}, t>0
$$

On suppose maintenant que

$$
|f(x)| \leq C\left(1+|x|^{q}\right),
$$

$q$ "assez petit" dans le cas $\alpha=-1$. Alors $f$ est $\mu$-intégrable, et on suppose enfin que

$$
\int_{\mathbb{R}^{d}} f(x) \mu(d x)=0
$$

Théorème 2.2 Sous les hypothèses (3),...,(6), l'intégrale dans la formule (2) converge, et la fonction ainsi définie est l'unique solution appartenant à $W_{l o c}^{2, p}\left(\mathbb{R}^{d}\right)$ pour tout $p>1$ et satisfaisant $\int u(x) \mu(d x)=0$ de l'équation (1).

La démonstration de ce théorème est essentiellement probabiliste, sauf en ce qui concerne l'appartenance à $W_{l o c}^{2, p}\left(\mathbb{R}^{d}\right)$, laquelle se démontre par comparaison avec la solution d'une EDP elliptique sur un ouvert borné. On démontre enfin par un argument probabiliste le :

Théorème 2.3 On suppose satisfaites les hypothèses du Théorème 2.2.

(i) Si en outre, pour certains $\beta<0$ et $C>0, x \in \mathbb{R}^{d}$,

$$
|f(x)| \leq C(1+|x|)^{\beta+\alpha-1},
$$

alors u est bornée, et

$$
|\nabla u(x)| \leq C^{\prime}(1+|x|)^{(\beta+\alpha-1)^{+}} .
$$

(ii) Si en outre il existe $\beta, C>0$ tels que

$$
|f(x)| \leq C(1+|x|)^{\beta+\alpha-1}
$$

avec aussi dans le cas $\alpha=-1$ et $\beta>4,2 r>\Lambda d+(\beta-2) \lambda_{+}$, alors

$$
\begin{aligned}
|u(x)| & \leq C^{\prime}\left(1+|x|^{\beta}\right), \\
|\nabla u(x)| & \leq C^{\prime}\left(1+|x|^{(\beta+\alpha-1)^{+}}+|x|^{\beta}\right) .
\end{aligned}
$$




\section{3 Équations différentielles stochastiques rétrogrades et sys- tèmes d'équations aux dérivées partielles semilinéaires}

Considérons l'EDP parabolique linéaire

$$
\left\{\begin{aligned}
\frac{\partial u}{\partial t}(t, x)+(L u)(t, x)+c(x) u(t, x) & =0,0<t<T ; x \in \mathbb{R}^{d} ; \\
u(T, x) & =g(x), x \in \mathbb{R}^{d}
\end{aligned}\right.
$$

où

$$
L=\frac{1}{2} \sum_{i, j}\left(\sigma \sigma^{*}\right)_{i j} \frac{\partial^{2}}{\partial x_{i} \partial y_{j}}+b_{i} \frac{\partial}{\partial x_{i}}
$$

est le générateur infinitésimal d'un processus de diffusion homogène $\left\{X_{t} ; t \geq 0\right\}$. Nous allons retrouver la formule de Feynman-Kac de l'introduction via la formule de TrotterKato.

Soit $h>0$ tel que $n=h^{-1}(T-t)$ est un entier, et

$$
t=t_{0}<t_{1}=t_{0}+h<\cdots<t_{n}=T \text {. }
$$

D’après la formule de Trotter-Kato, pour $h$ petit

$$
u(t, x) \simeq\left[T_{h} \circ e^{h c(\cdot)} \circ T_{h} \circ e^{h c(\cdot)} \circ \cdots \circ T_{h}\left(e^{h c(\cdot)} g\right)\right](x),
$$

où $\left\{T_{t} ; t \geq 0\right\}$ désigne le semigroupe de générateur infinitésimal $L, e^{h c(\cdot)}$ l'opérateur de multiplication par la fonction $e^{h c(x)}$, et l'opérateur $T_{h} \circ e^{h c(\cdot)}$ est appliqué $n$ fois. Notons que

$$
\begin{aligned}
\left(T_{h} f\right)(x) & =\mathbb{E}\left[f\left(X_{s+h}\right) / X_{s}=x\right] \\
\left(T_{h} f\right)\left(X_{s}\right) & =\mathbb{E}\left[f\left(X_{s+h} / X_{s}\right]\right. \\
& =\mathbb{E}\left[f\left(X_{s+h}\right) / \mathcal{F}_{s}^{X}\right],
\end{aligned}
$$

oì $\mathcal{F}_{s}^{X}$ est la "filtration naturelle" de $X$ définie par

$$
\mathcal{F}_{s}^{X}=\sigma\left\{X_{r} ; 0 \leq r \leq s\right\}
$$

et la dernière égalité exprime la propriété de Markov de $X$. Donc la formule approchée ci-dessus se réécrit :

$$
u(t, x) \simeq \mathbb{E}^{X_{t}=x}\left[e^{h c\left(X_{t_{1}}\right)} \mathbb{E}^{\mathcal{F}_{t_{1}}} e^{h c\left(X_{t_{2}}\right)} \cdots \mathbb{E}^{\mathcal{F}_{t_{n-1}}}\left(e^{h c\left(X_{T}\right)} g\left(X_{T}\right)\right)\right] .
$$

Il résulte des propriétés de l'espérance conditionnelle que

$$
u(t, x) \simeq \mathbb{E}^{X_{t}=x}\left[e^{h \sum_{i=1}^{n} c\left(X_{t_{i}}\right)} g\left(X_{T}\right)\right],
$$

et la limite quand $h \rightarrow 0$ donne

$$
u(t, x)=\mathbb{E}^{X_{t}=x}\left[e^{h \int_{t}^{T} c\left(X_{s}\right) d s} g\left(X_{T}\right)\right] .
$$


Nous allons maintenant tenter d'adopter la même démarche pour l'équation semilinéaire :

$$
\left\{\begin{aligned}
\frac{\partial u}{\partial t}(t, x)+(L u)(t, x)+f(u(t, x)) & =0,0<t<T, x \in \mathbb{R}^{d}, \\
u(T, x) & =g(x), x \in \mathbb{R}^{d} .
\end{aligned}\right.
$$

Pour $h>0, u \in \mathbb{R}$, soit $\Phi_{h}(u)$ la valeur à l'instant $h$ de la solution de l'EDO :

$$
\frac{d Y_{t}}{d t}=f\left(Y_{t}\right), Y_{0}=u
$$

La formule de Trotter-Kato appliquée à l'équation semilinéaire ci-dessus donne

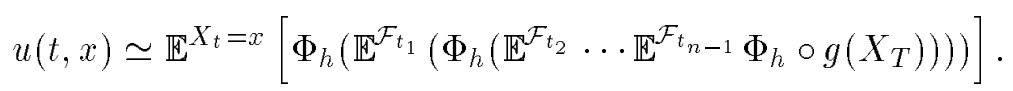

Quelle est la limite quand $h \rightarrow 0$ de cette formule?

Ce que l'on voudrait est une façon de calculer l'évolution de $u\left(t, X_{t}\right)$ en temps rétrograde, depuis $g\left(X_{T}\right)$ jusqu'à $u(t, x)$, qui ne fasse pas intervenir la connaissance de la fonction $\left\{u(s, y) ; 0 \leq s \leq T, y \in \mathbb{R}^{d}\right\}$. Notons que, à la limite quand $h \rightarrow 0$ dans la formule de Trotter-Kato, l'évolution de $u\left(t, X_{t}\right)$ combine deux effets : elle suit - en temps rétrograde - l'EDO de coefficient $-f$, et $u\left(t, X_{t}\right)$ est projetée - en temps continu sur la $\sigma$-algèbre $\mathcal{F}_{t}=\mathcal{F}_{t}^{X}$. Notons que la grosse différence avec le cas linéaire est que, puisque l'espérance conditionnelle ne commute pas avec l'opérateur $\Phi_{h}$, on ne peut pas espérer obtenir un algorithme qui calculerait une évolution trajectoire par trajectoire, pour finalement prendre une espérance.

Supposons que $\left\{Y_{t} ; 0 \leq t \leq T\right\}$ est solution de :

$$
Y_{t}=\mathbb{E}^{\mathcal{F}_{t}}\left[g\left(X_{T}\right)+\int_{t}^{T} f\left(Y_{s}\right) d s\right], 0 \leq t \leq T
$$

Alors $Y_{t}$ est un bon candidat pour être égal à $u\left(t, X_{t}\right)$. En effet, supposons que $u \in$ $C^{1,2}\left((0, T) \times \mathbb{R}^{d}\right)$ est une solution classique de l'EDP ci-dessus. Alors, par la formule d'Itô

$$
u\left(t, X_{t}\right)=u\left(T, X_{T}\right)-\int_{t}^{T}\left(\frac{\partial u}{\partial s}+L u\right)\left(s, X_{s}\right) d s-\left(M_{T}-M_{t}\right),
$$

où $\left\{M_{t}\right\}$ est une martingale locale.

En admettant que cette martingale locale est une martingale, et en utilisant l'équation satisfaite par $u$, on en déduit que

$$
u\left(t, X_{t}\right)=\mathbb{E}^{\mathcal{F}_{t}}\left[g\left(X_{T}\right)+\int_{t}^{T} f\left(u\left(s, X_{s}\right)\right) d s\right]
$$

ce qui a un sens en tout cas si $f$ et $g$ sont bornées. Donc l'équation différentielle stochastique rétrograde (7) a au moins la solution $Y_{t}=u\left(t, X_{t}\right)$. L'unicité dans la classe des processus satisfaisant $\mathbb{E}\left(\sup _{0 \leq t \leq T} Y_{t}^{2}\right)<\infty$ découle de l'hypothèse de Lipschitz. 
Nous allons maintenant réécrire l'équation différentielle stochastique rétrograde EDSR - (7) sous une autre forme. Supposons pour fixer les idées que $\left\{X_{t}\right\}$ est solution forte d'une EDS dirigée par le mouvement brownien $d$-dimensionnel $\left\{B_{t} ; t \geq 0\right\}$, si bien que $\left\{X_{t} ; 0 \leq t \leq T\right\}$ est une fonction de $\left\{B_{t} ; 0 \leq t \leq T\right\}$, et on peut choisir comme filtration la filtration naturelle de $B: \mathcal{F}_{t}=\mathcal{F}_{t}^{B}$. Considérons la variable aléatoire

$$
\xi=g\left(X_{T}\right)+\int_{0}^{T} f\left(Y_{t}\right) d t
$$

et supposons qu'elle est de carré intégrable; elle est $\mathcal{F}_{T}$ mesurable. Il résulte alors d'un célèbre théorème de représentation dî̀ à Itô qu'il existe un processus $d$-dimensionnel $\mathcal{F}_{t^{-}}$ adapté $\left\{Z_{t} ; 0 \leq t \leq T\right\}$ tel que

$$
\begin{aligned}
& \mathbb{E} \int_{0}^{T}\left|Z_{t}\right|^{2} d t<\infty, \\
& \xi=\mathbb{E}(\xi)+\int_{0}^{T}\left(Z_{t}, d B_{t}\right) .
\end{aligned}
$$

On constate aisément que $Y_{0}=\mathbb{E}^{\mathcal{F}_{0}}(\xi)=\mathbb{E}(\xi)$, soit

$$
Y_{0}=g\left(X_{T}\right)+\int_{0}^{T} f\left(Y_{t}\right) d t-\int_{0}^{T}\left(Z_{t}, d B_{t}\right)
$$

et la quantité

$$
Y_{0}-\int_{0}^{t} f\left(Y_{s}\right) d s+\int_{0}^{t}\left(Z_{s}, d B_{s}\right)
$$

est $\mathcal{F}_{t}$ mesurable et égale à

$$
g\left(X_{T}\right)+\int_{t}^{T} f\left(Y_{s}\right) d s-\int_{t}^{T}\left(Z_{s}, d B_{s}\right) .
$$

Donc d'après (7),

$$
Y_{t}=g\left(X_{T}\right)+\int_{t}^{T} f\left(Y_{s}\right) d s-\int_{t}^{T}\left(Z_{s}, d B_{s}\right) .
$$

Au point où nous sommes, pourquoi ne pas faire dépendre $f$ de $X_{s}$ et de $Z_{s}$, et rendre l'équation vectorielle si bien que le problème que nous cherchons à résoudre est le suivant. Etant donné $g: \mathbb{R}^{d} \rightarrow \mathbb{R}^{k}$ et $f: \mathbb{R}^{d} \times \mathbb{R}^{k} \times \mathbb{R}^{k \times d} \rightarrow \mathbb{R}^{k}$, trouver un couple $\left(Y_{t}, Z_{t}\right) \mathcal{F}_{t}$-adapté à valeurs dans $\mathbb{R}^{k} \times \mathbb{R}^{k \times d}$, tel que

$$
\begin{gathered}
\mathbb{E}\left(\sup _{0 \leq t \leq T}\left|Y_{t}\right|^{2}+\int_{0}^{T}\left\|Z_{s}\right\|^{2} d s\right)<\infty \\
Y_{t}=g\left(X_{T}\right)+\int_{t}^{T} f\left(X_{s}, Y_{s}, Z_{s}\right) d s-\int_{t}^{T} Z_{s} d B_{s}, \quad 0 \leq t \leq T
\end{gathered}
$$


Remarque 3.1 La terminologie "EDSR" est en fait impropre. On peut considérer qu'il s'agit plutôt d'un "problème inverse pour une EDS". En fait, la formulation ci-dessus est équivalente au problème suivant : trouver $y \in \mathbb{R}^{k}$ et $\left\{Z_{t}\right\}$ adapté à valeurs dans $\mathbb{R}^{k \times d}$ t.q. $\mathbb{E} \int_{0}^{T}\left\|Z_{t}\right\|^{2} d t<\infty$, tels que la solution $\left\{Y_{t} ; 0 \leq t \leq T\right\}$ de l'EDS :

$$
Y_{t}=y-\int_{0}^{t} f\left(X_{s}, Y_{s}, Z_{s}\right) d s+\int_{0}^{t} Z_{s} d B_{s}
$$

satisfasse $\mathbb{E}\left(\sup _{0 \leq t \leq T}\left|Y_{t}\right|^{2}\right)<\infty$ et la condition finale:

$$
Y_{T}=g\left(X_{T}\right)
$$

On fait les hypothèses suivantes sur le coefficient $f$ et la condition finale $g: f$ et $g$ sont continues et pour certains $K, \mu, p>0, \varphi: \mathbb{R}_{+} \rightarrow \mathbb{R}_{+}$continue et croissante,

$$
\begin{aligned}
|g(x)| & \leq K\left(1+|x|^{p}\right) \\
|f(x, y, z)| & \leq K\left(1+|x|^{p}+\varphi(|y|)+\|z\|\right) \\
\left(y-y^{\prime}, f(x, y, z)-f\left(x, y^{\prime}, z\right)\right) & \leq \mu\left|y-y^{\prime}\right|^{2} \\
\left|f(x, y, z)-f\left(x, y, z^{\prime}\right)\right| & \leq K \| z-z^{\prime}|| .
\end{aligned}
$$

On suppose par ailleurs les coefficients $b$ et $\sigma$ de l'EDS satisfaite par $\left\{X_{t}\right\}$ localement lipschitziens et à croissance au plus linéaire à l'infini. On a alors le

Théorème 3.2 Sous les hypothèses ci-dessus, l'EDSR (8)-(9) possède une solution unique.

Ce résultat - avec des hypothèses plus restrictives - a été établi par Pardoux, Peng [30]. Pour une démonstration sous les hypothèses ci-dessus, on pourra consulter Pardoux [28]. Nous allons indiquer la démonstration de ce théorème sous l'hypothèse supplémentaire :

$$
\left|f(x, y, z)-f\left(x, y^{\prime}, z\right)\right| \leq K\left|y-y^{\prime}\right| .
$$

Preuve (sous les hypothèses $(10),(11),(13),(14))$. On note $\mathcal{B}^{2}$ la classe des processus adaptés à valeurs dans $\mathbb{R}^{k} \times \mathbb{R}^{k \times d}$, qui vérifient $(8)$. Etant donné $(U, V) \in \mathcal{B}^{2}$, on définit $(X, Y)=\Phi(U, V)$ comme suit

$$
Y_{t}=\mathbb{E}\left[g\left(X_{T}\right)+\int_{t}^{T} f\left(X_{s}, U_{s}, V_{s}\right) d s \mid \mathcal{F}_{t}\right]
$$

et $\left\{Z_{t} ; 0 \leq t \leq T\right\}$ est donné par le théorème de représentation d'Itô appliqué au v.a.

$$
\xi+\int_{0}^{T} f\left(X_{s}, U_{s}, V_{s}\right) d s
$$

ESAIM: Proc., Vol. 6, SePtembre 1998, 91-109 
Alors

$$
Y_{t}=g\left(X_{T}\right)+\int_{t}^{T} f\left(X_{s}, U_{s}, V_{s}\right) d s-\int_{t}^{T} Z_{s} d B_{s} .
$$

Puisque une solution de (9) est un point fixe de $\Phi$ dans $\mathcal{B}^{2}$, il reste à montrer que $\Phi$ est une contraction stricte dans $\mathcal{B}^{2}$ muni d'une certaine norme. Il résulte de la formule d'Itô que si $\bar{U}=U-U^{\prime}, \bar{V}=V-V^{\prime},(Y, Z)=\Phi(U, V),\left(Y^{\prime}, Z^{\prime}\right)=\Phi\left(U^{\prime}, V^{\prime}\right),(\bar{Y}, \bar{Z})=\left(Y-Y^{\prime}, Z-Z^{\prime}\right)$, et $\gamma \in \mathbb{R}$,

$$
e^{\gamma t} \mathbb{E}\left|\bar{Y}_{t}\right|^{2}+\mathbb{E} \int_{t}^{T} e^{\gamma s}\left(\gamma\left|\bar{Y}_{s}\right|^{2}+\left\|\bar{Z}_{s}\right\|^{2}\right) d s=E \mathbb{E} \int_{t}^{T} e^{\gamma s}\left(\bar{Y}_{s}, f\left(X_{s}, U_{s}, V_{s}\right)-f\left(X_{s}, U_{s}^{\prime}, V_{s}^{\prime}\right)\right) d s
$$

Il résulte alors du caractère lipschitzien de $f$ et de l'inégalité de Cauchy-Schwarz que si $\gamma=1+4 K^{2}$

$$
\mathbb{E} \int_{0}^{T} e^{\gamma t}\left(\left|\bar{Y}_{t}\right|^{2}+\left\|\bar{Z}_{t}\right\|^{2} d t\right) \leq \frac{1}{2} \mathbb{E} \int_{0}^{T} e^{\gamma t}\left(\left|\bar{U}_{t}\right|^{2}+\left\|\bar{V}_{t}\right\|^{2}\right) d t
$$

CQFD.

Nous allons maintenant donner un théorème de comparaison dans le cas $k=1$.

Théorème 3.3 Soit $(f, g)$ et $\left(f^{\prime}, g^{\prime}\right)$ deux données satisfaisant les conditions (10)-(19), et désignons respectivement par $(Y, Z)$ et $\left(Y^{\prime}, Z^{\prime}\right)$ la solution de $(9)$ associée à $(f, g)$ et celle associée à $\left(f^{\prime}, g^{\prime}\right)$.

Si $g(x) \leq g^{\prime}(x), f(x, y, z) \leq f^{\prime}(x, y, z), \forall(x, y, z) \in \mathbb{R}^{d} \times \mathbb{R}^{k} \times \mathbb{R}^{d}$, alors $Y_{t} \leq Y_{t}^{\prime}$, $0 \leq t \leq T$. Si en outre $Y_{0}=Y_{0}^{\prime}$, alors $Y_{t}=Y_{t}^{\prime}, 0 \leq t \leq T$, p.s. En particulier, si $f\left(X_{t}, y, z\right)<f^{\prime}\left(X_{t}, y, z\right), \forall(y, z) \in \mathbb{R} \times \mathbb{R}^{d}$, sur un ensemble de $d t \times d \mathbb{P}$ mesure positive, alors $Y_{0}<Y_{0}^{\prime}$.

Nous admettrons ce théorème, dont la preuve repose sur la formule pour la solution des EDSR linéaires.

Nous allons maintenant préciser le lien entre l'EDSR (8) et le système suivant d'EDP semilinéaires.

$$
\left\{\begin{aligned}
\frac{\partial u_{i}}{\partial t}(t, x)+L u_{i}(t, x)+f_{i}(x, u(t, x),(\nabla u \sigma)(t, x)) & =0, i=1 \cdots k, x \in \mathbb{R}^{d}, 0 \leq t \leq T \\
u(T, x) & =g(x), x \in \mathbb{R}^{d} .
\end{aligned}\right.
$$

On suppose que

$$
L=\frac{1}{2} \sum_{i, j} a_{i_{j}}(x) \frac{\partial^{2}}{\partial x_{i} \partial x_{j}}+\sum_{i} b_{i}(x) \frac{\partial}{\partial x_{i}}
$$

est le générateur infinitésimal de la diffusion solution de l'EDS :

$$
d X_{t}=b\left(X_{t}\right) d t+\sigma\left(X_{t}\right) d B_{t}
$$


i.e. $a=\sigma \sigma^{*}$. On suppose par exemple que $b$ et $\sigma$ sont globalement lipschitziens. On note $\left\{X_{s}^{t, x} ; s \geq t\right\}$ la solution partant de $x$ à l'instant $t$, et $\left\{\left(Y_{s}^{t, x}, Z_{s}^{t, x}\right) ; t \leq s \leq T\right\}$ la solution de l'EDS rétrograde

$$
\left.Y_{s}^{t, x}=g\left(X_{T}^{t, x}\right)+\int_{s}^{T} f\left(X_{r}^{t, x}, Y_{r}^{t, x}, Z_{r}^{t, x}\right)\right) d r-\int_{s}^{T} Z_{r}^{t, x} d B_{r}
$$

qui est telle que $Y_{t}^{t, x}$ est déterministe ( $t$ joue ici le rôle de l'instant 0 dans $(9)$ ). On a le

Théorème 3.4 Soit $u \in C^{1,2}\left([0, T] \times \mathbb{R}^{d} ; \mathbb{R}^{k}\right)$ une solution classique du système d'équations (15), qui satisfait pour certains $c, q>0$,

$$
|u(t, x)|+\left|\nabla_{x} u(t, x)\right| \leq c\left(1+|x|^{q}\right) .
$$

Alors, pour tout $(t, x) \in[0, T] \times \mathbb{R}^{d},\left\{\left(u\left(s, X_{s}^{t, x}\right),(\nabla u \sigma)\left(s, X_{s}^{t, x}\right)\right) ; t \leq s \leq T\right\}$ est la solution de l'EDSR (16). En particulier, $u(t, x)=Y_{t}^{t, x}$.

Preuve La condition de l'énoncé entraîne que

$$
\mathbb{E}\left(\sup _{t \leq s \leq T}\left|u\left(s, X_{s}^{t, x}\right)\right|^{2}+\int_{t}^{T}\left\|\left(\nabla_{x} u \sigma\right)\left(s, X_{s}^{t, x}\right)\right\|^{2} d s\right)<\infty .
$$

Il reste à appliquer la formule d'Itô à $u\left(s, X_{s}^{t, x}\right)$.

Le résultat suivant dit que sous les hypothèses du Théorème $3.2, u(t, x) \triangleq Y_{t}^{t, x}$ est solution de viscosité du système d'EDP (15). Cependant, pour que la notion de solution de viscosité ait un sens, on est obligé de supposer que la $i$-ème coordonnée $f_{i}$ de la fonction $f$ ne dépend que de la $i$-ème ligne de la matrice $z$, ce qui fait que $f_{i}$ ne dépend que du gradient de $u_{i}$. Définissons la notion de sous-solution de viscosité (la notion de sur-solution se définit de façon analogue, et une solution est à la fois une sous et une sur-solution).

Définition $3.5 u \in C\left([0, T] \times \mathbb{R}^{d} ; \mathbb{R}^{k}\right)$ est appelée sous-solution de viscosité de (15) si $u_{i}(T, x) \leq g_{i}(x), \forall 1 \leq i \leq k$, et pour tout $1 \leq i \leq k, \varphi \in C^{1,2}\left([0, T] \times \mathbb{R}^{d}\right)$ et $(t, x) \in$ $\left[0, T\left[\times \mathbb{R}^{d}\right.\right.$ qui est un minimum local de $u_{i}-\varphi$,

$$
-\frac{\partial \varphi}{\partial t}(t, x)-L \varphi(t, x)-f_{i}(x, u(t, x),(\nabla \varphi \sigma)(t, x)) \leq 0 .
$$

Théorème $3.6 u(t, x) \triangleq Y_{t}^{t, x}$ est continue, et elle est solution de viscosité du système d'EDP (15). 
Preuve Nous ne démontrerons pas la continuité. Montrons que $u$ est une sous-solution. Soit $1 \leq i \leq k, \varphi \in C^{1,2}\left([0, T] \times \mathbb{R}^{d} ; \mathbb{R}^{k}\right)$, et $(t, x) \in[0, T] \times \mathbb{R}^{d}$ qui est un maximum local de $u_{i}-\varphi$. On suppose par exemple que $u_{i}(t, x)=\varphi(t, x)$, et que $\forall t \leq s \leq t+\alpha$, $|y-x| \leq \alpha, u_{i}(s, y) \leq \varphi(s, y)$. On va supposer par l'absurde que pour les mêmes $(s, y)$ il suffit de choisir $\alpha$ suffisamment petit :

$$
\frac{\partial \varphi}{\partial t}+(L \varphi)(s, y)+f_{i}(s, y, u(s, y),(\nabla \varphi \sigma)(s, y))<0
$$

Posons

$$
\begin{aligned}
& \tau(\omega) \quad=\inf \left\{s \geq t ;\left|X_{s}^{t, x}(\omega)-x\right| \geq \alpha\right\} \wedge(t+\alpha) \\
& \left(\bar{Y}_{s}, \bar{Z}_{s}\right)=\left(\left(Y_{s \wedge \tau}^{t, x}\right)^{i}, \mathbf{1}_{[0, \tau]}(s)\left(Z_{s}^{t, x}\right)^{i}\right), t \leq s \leq t+\alpha \\
& \left(\hat{Y}_{s}, \hat{Z}_{s}\right)=\left(\varphi\left(s, X_{s \wedge \tau}^{t, x}\right), \mathbf{1}_{[0, \tau]}(s)(\nabla \varphi \sigma)\left(s, X_{s}^{t, x}\right)\right), t \leq s \leq t+\alpha .
\end{aligned}
$$

On remarque que ces processus sont respectivement solution de (la seconde EDSR résulte de la formule d'Itô, et le fait que $\bar{Y}_{\tau}=u_{i}\left(\tau, X_{\tau}^{t, x}\right)$ est une conséquence de la relation $Y_{t+h}^{t, x}=Y_{t+h}^{t+h, X_{t+h}^{t, x}} h>0$, qui résulte de l'unicité de la solution de l'EDSR (16)) :

$$
\begin{aligned}
\bar{Y}_{s}= & \left.u_{i}\left(\tau, X_{\tau}^{t, x}\right)+\int_{s}^{t+\alpha} \mathbf{1}_{[0, \tau]}(r) f_{i}\left(X_{r}^{t, x}, u k, X_{r}^{t, x}\right), \bar{Z}_{r}\right) d r \\
& -\int_{s}^{t+\alpha} \bar{Z}_{r} d B_{r}, \quad t \leq s \leq t+\alpha \\
\bar{Y}_{s}= & \varphi\left(\tau, X_{\tau}^{t, x}\right)-\int_{s}^{t+\alpha} \mathbf{1}_{[0, \tau]}(r)\left(\frac{\partial \varphi}{\partial r}+L \varphi\right)\left(r, X_{r}^{t, x}\right) d r \\
& -\int_{s}^{t+\alpha} \hat{Z}_{r} d B_{r}, t \leq s \leq t+\alpha
\end{aligned}
$$

le théorème de comparaison 3.3 entraîne, d'après l'hypothèse faite sur $\varphi, \bar{Y}_{t}<\hat{Y}_{t}$, soit $u_{i}(t, x)<\varphi(t, x)$, ce qui est absurde.

Remarque 3.7 Si l'on pose $v(t, x)=u(T-t, x)$, alors on a une formule probabiliste pour le système d'EDP paraboliques progressives

$$
\left\{\begin{aligned}
\frac{\partial v}{\partial t}(t, x) & =L v(t, x)+f(x, v(t, x),(\nabla v \sigma)(t, x)), t>0, x \in \mathbb{R}^{d} \\
v(0, x) & =g(x), x \in \mathbb{R}^{d}
\end{aligned}\right.
$$

en terme de la solution de l'EDSR

$$
\bar{Y}_{s}^{t, x}=g\left(X_{t}^{0, x}\right)+\int_{s}^{t} f\left(X_{r}^{0, x}, \bar{Y}_{r}^{t, x}, \bar{Z}_{r}^{t, x}\right) d r-\int_{s}^{t} \bar{Z}_{r}^{t, x} d B_{r}, 0 \leq s \leq t,
$$

au sens où $v(t, x)=\bar{Y}_{0}^{t, x}$. 
Remarque 3.8 Notons que l'on peut considérer des conditions au bord de type Dirichlet, ainsi que des conditions de Neumann non linéaires (cf. Pardoux, Zhang [33]).

On a également des formules probabilistes pour des systèmes d'EDP elliptiques.

Signalons encore deux extensions des résultats ci-dessus. D'une part, on peut considérer un système d'EDP du second ordre où l'opérateur linéaire $L$ qui apparaît est différent sur chaque ligne, cf. Pardoux [27]

Enfin, on peut traiter des équations quasilinéaires, en faisant dépendre les coefficients de l'équation de $X$ de la solution $(Y, Z)$ de l'EDSR, cf. Pardoux, Tang [31].

\section{Propagation d'onde dans les équations de réaction-diffusion.}

Les équations renormalisées de Kolmogorov, Petrovskii, Piskunov s'écrivent sous la forme

$$
\left\{\begin{aligned}
\frac{\partial u^{\varepsilon}}{\partial t}(t, x) & =\varepsilon L u^{\varepsilon}(t, x)+\frac{1}{\varepsilon} c(x) u^{\varepsilon}(t, x)\left(1-u^{\varepsilon}(t, x)\right), t>0, x \in \mathbb{R}^{d} \\
u^{\varepsilon}(0, x) & =g(x), x \in \mathbb{R}^{d}
\end{aligned}\right.
$$

avec $L$ opérateur elliptique, $0<\underline{c} \leq c(x) \leq \bar{c}, g$ est positive bornée. Freidlin [17] a montré à l'aide de techniques de grandes déviations que pour tout $x \in \mathbb{R}^{d}$, il existe une valeur critique $t_{x}<\infty$ telle que si $t<t_{x} u^{\varepsilon}(t, x)$ tend vers 0 , et si $t>t_{x}, u^{\varepsilon}(t, x)$ tend vers

1 , quand $\varepsilon$ tend vers 0. Evans, Souganidis [14] et Barles, Souganidis [1] ont précisé ce résultat en étudiant, à l'aide de techniques de solutions de viscosité, la convergence de $\varepsilon \log u^{\varepsilon}(t, x)$ vers une fonction $V \leq 0$.

Cela permet d'établir une vitesse de convergence exponentielle vers 0 , sur l'ensemble $V<0$. La vitesse de convergence vers 1 s'étudie en calculant la limite de $\varepsilon \log \left(1-u^{\varepsilon}(t, x)\right)$.

Pradeilles [36] généralise ces résultats à l'aide des EDSR dans deux directions. D'une part, il remplace l'hypothèse d'ellipticité par une hypothèse d'hypoellipticité. D'autre part, il considère des nonlinéarités dépendant du gradient de la solution. Enfin, dans [35], il étudie le cas des systèmes d'équations. Il semble que l'intérêt des EDSR réside - pour cet exemple - dans la capacité de contrôler $\nabla u^{\varepsilon}$ par $u^{\varepsilon}$.

\section{Homogénéisation d'EDP semilinéaires à coefficients périodiques}

Nous allons énoncer deux résultats en homogénéisation des EDP semilinéaires à coefficients périodiques, qui ont été démontrés récemment à l'aide des EDSR.

\section{1}

Le premier de ces résultats, dû à Buckdahn, Hu, Peng [8], traite l'homogénéisation du système d'EDP semilinéaires paraboliques 


$$
\left\{\begin{array}{l}
\frac{\partial u^{\varepsilon}}{\partial t}=L^{\varepsilon} u_{i}^{\varepsilon}(t, x)+f_{i}\left(x, \frac{x}{\varepsilon}, u^{\varepsilon}(t, x),\left(\nabla u_{i}^{\varepsilon} \sigma\right)(t, x), 1 \leq i \leq k,\right. \\
u^{\varepsilon}(0, x)=u_{0}(x)
\end{array}\right.
$$

où $L^{\varepsilon}=\frac{1}{2} \sum_{i, j} a_{i j}\left(\frac{x}{\varepsilon}\right) \frac{\partial^{2}}{\partial x_{i} \partial d_{j}}+\frac{1}{\varepsilon} \sum_{i}\left(\frac{x}{\varepsilon}\right) \frac{\partial}{\partial x_{1}}, a=\sigma \sigma^{*}, \sigma$ et $b$ sont périodiques de période un dans chaque direction et globalement lipschitziens, $a(x) \geq \alpha I>0, \forall x \in \mathbb{R}^{d}$. On suppose $f$ et $u_{0}$ bornés et uniformément continus, et $f(x, y, z)$ globalement lipschitzienne en $(y, z)$, uniformément par rapport à $x$.

Soit $\mu(d x)=p(x) d x$ l'unique probabilité invariante du processus $\left\{U_{t} ; t \geq 0\right\}$ sur le tore $\mathbb{T}^{d}$, solution de l'EDS :

$$
d U_{t}=b\left(U_{t}\right) d t+\sigma\left(U_{t}\right) d B_{t} ; t \geq 0 .
$$

De façon équivalente on peut définir $p$ comme l'unique solution positive de l'EDP sur le tore $\mathbb{T}^{d}$

$$
\left(L^{1}\right)^{*} p(x)=0
$$

qui vérifie $\int_{\mathbb{T} d} p(x) d x=1$. On suppose satisfaite l'hypothèse de centrage

$$
\int b_{i}(x) \mu(d x)=0,1 \leq i \leq d .
$$

Sous cette hypothèse, pour chaque $i$ il existe une unique solution dans $W^{2, p}\left(\mathbb{T}^{d}\right), \mu$ centrée, de l'équation de Poisson

$$
L \hat{b}_{i}(x)+b_{i}(x)=0,1 \leq i \leq d,
$$

qui est donnée par la formule

$$
\hat{b}_{i}(x)=\int_{0}^{\infty} \mathbb{E}_{x}\left[b_{i}\left(U_{t}\right)\right] d t
$$

cf. ci-dessus section 2 .

On définit alors les coefficients de l'équation homogénéisée :

$$
\begin{aligned}
A & =\int_{\mathbb{T}^{d}}(I+\nabla \hat{b}) a(I+\nabla \hat{b})^{*}(x) \mu(d x) \\
\bar{f}(x, y, z) & =\int_{\mathbb{T}^{d}} f\left(x, y, z(I+\nabla \hat{b})^{*}\left(x^{\prime}\right)\right) \mu\left(d x^{\prime}\right)
\end{aligned}
$$

On définit $u \in C_{b}\left([0, T] \times \mathbb{R}^{d} ; \mathbb{R}^{k}\right)$ comme la solution du système d'EDP homogénéisées :

$$
\left\{\begin{aligned}
\frac{\partial u_{\ell}}{\partial t}(t, x) & =\frac{1}{2} \sum_{i, j} A_{i j} u_{\ell}(t, x)+\bar{f}_{\ell}\left(x, u(t, x), \nabla u_{\ell}(t, x)(\bar{a})^{1 / 2}\right), 1 \leq \ell \leq k, t>0, x \in \mathbb{R}^{d} ; \\
u(0, x) & =u_{0}(x)
\end{aligned}\right.
$$

et on a le résultat : $u_{\varepsilon}(t, x) \rightarrow u(t, x), \forall t \geq 0, x \in \mathbb{R}^{d}$. 


\section{2}

Le second résultat, dû̀ à Pardoux [29], considère une EDP semilinéaire où la nonlinéarité ne peut pas dépendre du gradient, mais on a une nonlinéarité fortement oscillante. L'EDP à homogénéiser s'écrit :

$$
\left\{\begin{aligned}
\frac{\partial u^{\varepsilon}}{\partial t}(t, x) & =L^{\varepsilon} u^{\varepsilon}(t, x)+\frac{1}{\varepsilon} e\left(\frac{x}{\varepsilon}, u^{\varepsilon}(t, x)\right)+f\left(\frac{x}{\varepsilon}, u^{\varepsilon}(t, x)\right) \\
u^{\varepsilon}(0, x) & =u_{0}(x)
\end{aligned}\right.
$$

On suppose que

$$
L^{\varepsilon}=\frac{1}{2} \sum_{i, j} a_{i j}\left(\frac{x}{\varepsilon}\right) \frac{\partial^{2}}{\partial x_{i} \partial x_{j}}+\frac{1}{\varepsilon} \sum_{i} b_{i}\left(\frac{x}{\varepsilon}\right) \frac{\partial}{\partial x_{i}}+\sum_{i} c_{i}\left(\frac{x}{\varepsilon}\right) \frac{\partial}{\partial x_{i}}
$$

avec $a$ continue, $b$ et $c$ mesurables bornés, et les trois coefficients sont périodiques de période 1 dans chaque direction, $a(x) \geq \alpha I>0, a_{i j}=a_{j i}$, et $\sum_{j=1}^{d} \frac{\partial a_{i j}}{\partial x_{j}}$ est une fonction bornée, $1 \leq i \leq k$. On suppose à nouveau que

$$
\int_{\mathbb{T}^{d}} b_{i}(x) \mu(d x)=0,1 \leq i \leq k
$$

et aussi que

$$
\int_{\mathbb{T}^{d}} e(x, y) \mu(d x)=0, y \in \mathbb{R}
$$

où $\mu$ est la mesure invariante de la section 5.1. On suppose en outre que $e$ est de classe $C_{b}^{2}$ en $y$, uniformément par rapport à $x$.

Enfin on suppose que $f$ est mesurable et vérifie

$$
\begin{aligned}
\left(f(x, y)-f\left(x, y^{\prime}\right)\right)\left(y-y^{\prime}\right) & \leq \mu\left|y-y^{\prime}\right|^{2} \\
|f(x, y)| & \leq K\left(1+|y|^{2}\right),
\end{aligned}
$$

pour certains $\mu, K$ et tous les $x, y, y^{\prime}$.

Enfin, $u_{0}$ est supposée continue à croissance polynomiale à l'infini.

Outre les fonctions $\hat{b}_{i}, 1 \leq i \leq k$ de la section 5.1 , on définit pour chaque $y \in \mathbb{R}$ la solution de l'équation de Poisson sur $\mathbb{T}^{d}$ :

$$
L \hat{e}(x, y)+e(x, y)=0 .
$$

Sous des conditions supplémentaires assurant que la solution de l'EDP (19) est unique et "coïncide avec la solution de l'EDSR correspondante", on a $u_{\varepsilon}(t, x) \rightarrow u(t, x)$, où $u$ est la solution de l'EDP semilinéaire :

$$
\left\{\begin{aligned}
\frac{\partial u}{\partial t}(t, x) & =\frac{1}{2} \sum_{i, j} A_{i j} \frac{\partial^{2} u}{\partial x_{i} \partial x_{j}}(t, x)+\sum_{i=1}^{d} C_{i}(u(t, x)) \frac{\partial u}{\partial x_{i}}(t, x)+D(u(t, x)), \\
u(0, x) & =u_{0}(x)
\end{aligned}\right.
$$


et

$$
\begin{array}{ll}
A & =\int_{\mathbb{T}^{d}}(I+\nabla \hat{b}) a(I+\nabla \hat{b})^{*}(x) \mu(d x) \\
C(y) & \left.=\int_{\mathbb{T}^{d}}\left[(I+\nabla \hat{b}) c+a \frac{\partial^{2} \hat{e}^{*}}{\partial x, \partial y}(\cdot, y)\right)\right](x) \mu(d x) \\
D(y) & =\int_{\mathbb{T}^{d}}\left[<\frac{\partial \hat{e}}{\partial x}(\cdot, y), c>-\frac{\partial \hat{\hat{e}}}{\partial y} e(\cdot, y)+\frac{\partial^{2} \hat{e}}{\partial x \partial y} a \frac{\partial \hat{e}^{*}}{\partial x}(\cdot, y)+f(\cdot, y)\right](x) \mu(d x) .
\end{array}
$$

Remarque 5.1 On trouvera d'autres résultats d'homogénéisation d'EDP non linéaires, y compris d'homogénéisation stochastique (au lieu de périodique), démontrés par des méthodes analogues, dans Gaudron, Pardoux [18]. Voir aussi Briand, Hu, [7], Hu, Peng [19].

\section{Références}

[1] G. Barles, P.E. Souganidis. A remark on the asymptotic behavior of the solution of the KPP equation. C. R. Acad. Sci. Paris, 319, 1994, 679-684.

[2] R. BAss. Probabilistic techniques in analysis. Springer, 1995.

[3] R. BAss. Diffusions and elliptic operators. Probability and its Applications, Springer, 1998.

[4] S. Benachour, P. Chassaing, B. Roynette, P. Vallois. Processus associés à l'équation des milieux poreux. Ann. Scuola Norm. Sup. Pisa Cl. Sci., 23, 1997, 793-832.

[5] A. Bensoussan, J.L. Lions. Application des inéquations variationnelles en contrôle stochastique. Dunod, 1978.

[6] A. Bensoussan, J.L. Lions. Contrôle impulsionnel et inéquations quasi-variationnelles. Dunod, 1981.

[7] P. Briand, Y. Hu. Stability of BSDEs with random terminal time and homogenization of semilinear elliptic PDEs. J. Funct. Anal., 155, 1998, 455-494.

[8] R. Buckdahn, Y. Hu, S. Peng. Probabilistic approach to homogenization of viscosity solutions of parabolic PDEs. Nonlinear differential equations and Applications, à paraître.

[9] R. Dautray et al. Méthodes probabilistes pour les équations de la physique. Eyrolles, 1989.

[10] L. Desvillettes, C. Graham, S. Méléard. Probabilistic interpretation and numerical approximation of a Kac equation without cutoff. Soumis.

[11] J.S. Dhersin, J.F. Le Gall. Wiener's test for super-Brownian motion and the Brownian snake. Probab. Theory and Rel. Fields, 108, 1997, 103-129.

[12] E.B. Dynkin. Superprocesses and partial differential equations. Annals of Probab., 21, 1993, $1185-1262$.

[13] E. B. Dynkin, S. E. Kuznetsov. Fine topology and fine trace on the boundary associated with a class of semilinear differential equations. Comm. Pure Appl. Math., 51, 1998, 897-936.

[14] L.C. Evans, P.E. Souganidis. A PDE approach to geometric optics for certain semilinear parabolic equations. Indiana Univ. Math. J., 38, 1989, 141-172.

[15] W.H. Fleming, H.M. Soner. Controlled Markov processes and viscosity solutions. Applications of Mathematics, Springer, 1993. 
[16] M. FreidLin. Functional integration and partial differential equations. Princeton University Press, 1985.

[17] M. Freidinin. Markov processes and differential equations : asymptotic problems. Lecture Notes in Mathematics -EHT Zürich, Birkhäuser, 1996.

[18] G. Gaudron, É. Pardoux. EDSR convergence en loi et homogénéisation d'EDP semilinéaires. En préparation.

[19] Y. Hu, S. PENG. A stability theorem of backward stochastic differential equations and its applications. C. R. Acad. Sci. Paris, 324-9, 1997, 1059-1064.

[20] B. Lapeyre, É. Pardoux, R. Sentis. Méthodes de Monte-Carlo pour les équations de transport et de diffusion. Mathématiques et Applications 29, Springer, 1997.

[21] J. F. LE GALL. A path-valued Markov process and its connections with partial differential equations. In First European Congress of Mathematics, Vol. II, Birkhäuser, 1994.

[22] J. F. LE Gall. A probabilistic Poisson representation for positive solutions of $\Delta u=u^{2}$. Comm. Pure and Applied Math., 50, 1997, 69-103.

[23] Y. Le Jan, A.S. Sznitman. Stochastic cascades and 3-dimensional Navier-Stokes equations. Proba. Th. Relat. Fields, 109, 1997, 343-366.

[24] H. P. Mackean. A class of Markov processes associated with nonlinear parabolic equations. Proc. Nat. Acad. Sci, 56, 1966, 1907-1911.

[25] S. MÉLÉARD. Probabilistic interpretation and approximations of some Boltzmann equations. In Ve Simposio de Probabilidad y Processos Estocasticos, Guanajiato, 1998.

[26] S. MÉLÉARD. A probabilistic proof of the vortex method for the $2 \mathrm{~d}$ Navier-Stokes equation. Soumis.

[27] É. PARDoux. Backward stochastic differential equations and viscosity solutions of systems of semilinear parabolic and elliptic PDEs of second order. In L. Decreusefond, J. GJerde, B. Øksendal, A.S. Üstünel, editors, Stochastic Analysis and Related Topics VI: The Geilo Workshop, 1996, Birkhäuser, 1998, 79-127.

[28] É. PARdoux. BSDE's weak convergence and homogenization of semilinear PDE's. In S.M.S. Montréal 1998, à paraitre.

[29] É. Pardoux. Homogenization of linear and semilinear second order PDEs with periodic coefficients : a probabilistic approach. Soumis.

[30] É. Pardoux, S. Peng. Adapted solution of a backward stochastic differential equation. Systems \& Control letters, 14, 1990, 55-61.

[31] É. Pardoux, S. Tang. Forward-backward stochastic differential equations and quasilinear parabolic PDEs. Probab. Theory Relat. Fields, à paraître.

[32] É. Pardoux, A. Yu. Veretennikov. On Poisson equation and diffusion approximation 1. Soumis.

[33] É. Pardoux, S. Zhang. Generalized BSDEs and nonlinear Neumann boundary value problems. Prob. Theory and Rel. Fields, 110, 1998, 535-558.

[34] S.C. Port, C.J. Stone Brownian motion and classical potential theory. Acad. Press, 1978. 
[35] F. Pradeilles. Wave front propagation for reaction-diffusion systems and backward SDE's. Annals of Prob., à paraître.

[36] F. Pradeilles. Wave front propagation for generalized KPP equations. Soumis.

[37] A.S. Sznitman. Topics in propagation of chaos. In Ecole d'été de Probabilité de St Flour XIX. Lecture Notes in Mathematics 1464, Springer, 1991.

[38] A. Yu. Veretennikov. On polynomial mixing bounds for stochastic differential equations. Stoch. Proc. Appl., 70, 1997, 115-127.

LATP-UMR CNRS 6632

Centre de Mathématiques et Informatique

Université de Provence

39 , rue F. Joliot-Curie

13453 Marseille cedex 13

http://protis.univ-mrs.fr/LATP/EP/pardoux.html 